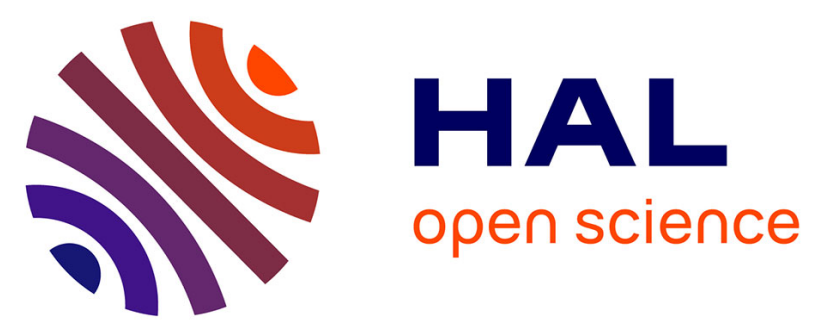

\title{
Health-Related Quality of Life and Physical Activity in Children with Inherited Cardiac Arrhythmia or Inherited Cardiomyopathies: The Prospective Multicentre Controlled QUALIMYORYTHM Study Rationale, Design and Methods.
}

Pascal Amedro, Oscar Werner, Hamouda Abassi, Aymeric Boisson, Luc Souilla, Sophie Guillaumont, Johanna Calderon, Stefan Matecki, Gregoire de La Villeon, Anne Requirand, et al.

\section{To cite this version:}

Pascal Amedro, Oscar Werner, Hamouda Abassi, Aymeric Boisson, Luc Souilla, et al.. Health-Related Quality of Life and Physical Activity in Children with Inherited Cardiac Arrhythmia or Inherited Cardiomyopathies: The Prospective Multicentre Controlled QUALIMYORYTHM Study Rationale, Design and Methods.. 2021. hal-03202623

\section{HAL Id: hal-03202623 https://hal.science/hal-03202623}

Preprint submitted on 12 Jun 2021

HAL is a multi-disciplinary open access archive for the deposit and dissemination of scientific research documents, whether they are published or not. The documents may come from teaching and research institutions in France or abroad, or from public or private research centers.
L'archive ouverte pluridisciplinaire HAL, est destinée au dépôt et à la diffusion de documents scientifiques de niveau recherche, publiés ou non, émanant des établissements d'enseignement et de recherche français ou étrangers, des laboratoires publics ou privés. 


\section{Health-Related Quality of Life and Physical Activity in Children with Inherited Cardiac Arrhythmia or Inherited Cardiomyopathies: The Prospective Multicentre Controlled QUALIMYORYTHM Study Rationale, Design and Methods.}

Pascal Amedro ( $\nabla$ pascal.amedro@gmail.com )

Montpellier University Hospital https://orcid.org/0000-0003-3649-0294

\section{Oscar Werner}

CHU Montpellier: Centre Hospitalier Regional Universitaire de Montpellier Hamouda Abassi

CHU Montpellier: Centre Hospitalier Regional Universitaire de Montpellier Aymeric Boisson

CHU Montpellier: Centre Hospitalier Regional Universitaire de Montpellier Luc Souilla

CHU Montpellier: Centre Hospitalier Regional Universitaire de Montpellier Sophie Guillaumont

CHU Montpellier: Centre Hospitalier Regional Universitaire de Montpellier Johanna Calderon

CHU Montpellier: Centre Hospitalier Regional Universitaire de Montpellier Stefan Matecki

CHU Montpellier: Centre Hospitalier Regional Universitaire de Montpellier Gregoire De La Villeon

CHU Montpellier: Centre Hospitalier Regional Universitaire de Montpellier

Anne Requirand

CHU Montpellier: Centre Hospitalier Regional Universitaire de Montpellier

Marie Vincenti

CHU Montpellier: Centre Hospitalier Regional Universitaire de Montpellier

Victor Pommier

CHU Montpellier: Centre Hospitalier Regional Universitaire de Montpellier

Kathleen Lavastre

CHU Montpellier: Centre Hospitalier Regional Universitaire de Montpellier

Alain Lacampagne

Montpellier University: Universite de Montpellier 


\section{Marie-Christine Picot}

CHU Montpellier: Centre Hospitalier Regional Universitaire de Montpellier

\section{Constance Beyler}

APHP: Assistance Publique - Hopitaux de Paris

\section{Christophe Delclaux}

APHP: Assistance Publique - Hopitaux de Paris

\section{Yves Dulac}

CHU Toulouse: Centre Hospitalier Universitaire de Toulouse

\section{Aitor Guitarte}

CHU Toulouse: Centre Hospitalier Universitaire de Toulouse

\section{Philippe Charron}

APHP: Assistance Publique - Hopitaux de Paris

\section{Isabelle Denjoy-Urbain}

APHP: Assistance Publique - Hopitaux de Paris

\section{Vincent Probst}

CHU Nantes: Centre Hospitalier Universitaire de Nantes

\section{Alban-Elouen Baruteau}

CHU Nantes: Centre Hospitalier Universitaire de Nantes

\section{Philippe Chevalier}

CHU Lyon: Hospices Civils de Lyon

\section{Sylvie Di Filippo}

CHU Lyon: Hospices Civils de Lyon

\section{Jean-Benoit Thambo}

CHU de Bordeaux Hôpital Cardiologique: Centre Hospitalier Universitaire de Bordeaux Hopital Cardiologique

\section{Damien Bonnet}

APHP: Assistance Publique - Hopitaux de Paris

\section{Jean-Luc Pasquie}

CHU Montpellier: Centre Hospitalier Regional Universitaire de Montpellier

\section{Study protocol}

Keywords: quality of life, physical activity, paediatrics, inherited cardiac arrhythmia, genetic cardiomyopathies

DOI: https://doi.org/10.21203/rs.3.rs-410935/v1

License: (c) (1) This work is licensed under a Creative Commons Attribution 4.0 International License. Read Full License 


\section{Abstract}

Background. Advances in paediatric cardiology has improved the prognosis of children with inherited cardiac disorders. However, health-related quality of life (QoL) and physical activity have been scarcely analysed in children with inherited cardiac arrhythmia or inherited cardiomyopathies. Moreover, current guidelines on the eligibility of young athletes with inherited cardiac disorders for sports participation mainly rely on expert opinions and remain controversial.

Methods. The QUALIMYORYTHM trial is a multicentre observational controlled study. The main objective is to compare the QoL of children aged 6 to 18 years old with inherited cardiac arrhythmia (long QT syndrome, Brugada syndrome, catecholaminergic polymorphic ventricular tachycardia, or arrhythmogenic right ventricular dysplasia), or inherited cardiomyopathies (hypertrophic, dilated, or restrictive cardiomyopathy), to that of age and gender-matched healthy subjects. The secondary objective is to assess their QoL according to the disease's clinical and genetic characteristics, the level of physical activity and motivation for sports, the exercise capacity, and the socio-demographic data. Participants will wear a fitness tracker (ActiGraph GT3X accelerometer) for 2 weeks. A total of 214 children are required to observe a significant difference of $7 \pm 15$ points in the PedsQL, with a power of $90 \%$ and an alpha risk of $5 \%$.

Discussion. After focusing on the survival in children with inherited cardiac disorders, current research is expanding to patient-reported outcomes and secondary prevention. The QUALIMYORYTHM trial intends to improve the level of evidence for future guidelines on sports eligibility in this population.

Trial registration. ClinicalTrials.gov Identifier: NCT04712136, registered on January 15th, 2021 (https://clinicaltrials.gov/ct2/show/NCT04712136).

\section{Background}

Prognosis of children with congenital cardiac disorders has significantly improved in the past two decades, due to great advances in prenatal screening, intensive neonatal care and modern invasive therapies [1]. As a result, more attention has been recently given to health-related quality of life (QoL) and secondary prevention in paediatric cardiology $[2,3]$.

Traditionally, congenital cardiac disorders are divided into 3 groups: congenital heart disease (CHD) for structural cardiac anomalies, inherited cardiac arrhythmia and inherited cardiomyopathies. So far, QoL studies in paediatric cardiology have mainly focused on the group of children with $\mathrm{CHD}$ and, although the overall level of QoL is good in this population, our group highlighted the existing correlation between exercise capacity and QoL, and the risk for physical deconditioning, even for non-serious CHD $[4,5]$. Indeed, despite the promotion of physical activity in the current guidelines [6-8], many children with CHD experience social barriers to physical activity and "remain on the side-line" at school or in their social life [9]. Ultimately, these patients may be exposed to the consequences of a sedentary lifestyle: overweight, 
high blood pressure, social exclusion, and impaired QoL [10-12]. Conversely, patients with CHD who have been physically active since childhood are less likely to become sedentary adults [13].

Such consistent data are not available in the literature for children from the two other groups, e.g. inherited cardiac arrhythmia or inherited cardiomyopathies, for which QoL and physical activity have been rarely studied. Some heterogeneous data were provided from small paediatric [14-16] or adult [17, 18] cohorts, however no prospective controlled study has analysed the determinants of QoL and the level of physical activity in children with inherited cardiac disorders [19].

The multicentre observational controlled QUALIMYORYTHM trial aims to evaluate the QoL, exercise capacity, and the level of physical activity of children with inherited cardiac arrhythmia or inherited cardiomyopathies, in comparison with a matched control population. This study will also evaluate the main factors associated with QoL in this population.

\section{Methods}

\subsection{Objectives}

The main objective of the QUALIMYORYTHM trial is to compare the QoL of children aged 6 to 18 years old with inherited cardiac arrhythmia or inherited cardiomyopathies to that of matched healthy children.

The secondary objective is to assess, in this population, the QoL according to the disease clinical and genetic characteristics, the level of physical activity, the exercise capacity, and the socio-demographic data.

\subsection{Study design}

The QUALIMYORYTHM trial is a prospective, multicentre controlled observational study with 1-month follow-up and 18-month recruitment duration. All screened subjects will be identifiable throughout the study by a unique subject number. The eligible patients will be divided into 2 groups (Fig. 1):

Group 1: children aged 6 to 18 years old with an inherited cardiac arrhythmia or an inherited cardiomyopathy.

Group 2: age and gender-matched control group, with healthy children aged 6 to 18 years old.

\subsection{Setting}

Patients will be recruited during a paediatric cardiology consultation among the French expert centres for inherited cardiac arrhythmia or an inherited cardiomyopathies (CARDIOGEN national network, http://www.filiere-cardiogen.fr). Overall, 7 university hospitals, labelled as expert centres in paediatric inherited cardiac disorders by health authorities, will be in charge of patient recruitment. 
Conduct of the study will be led by the principal investigator (supported, when necessary, by the coinvestigators), a research fellow, and a clinical research coordinator, all of whom are trained in Good Clinical Practice and in the requirements of the study protocol.

\subsection{Study population}

Children aged of 6 to 18 years old with an inherited cardiac arrhythmia (long QT syndrome, Brugada syndrome, catecholaminergic polymorphic ventricular tachycardia, or arrhythmogenic right ventricular dysplasia), or those with an inherited cardiomyopathy (hypertrophic, dilated, or restrictive cardiomyopathy), will be prospectively recruited during their annual follow-up.

The age- and gender-matched control group will be defined using the same method as in our previous paediatric exercise test studies [4, 5, 20-22]. Children aged 6 to 18 years old referred to the paediatric cardiology consultation for a non-severe functional symptom linked to exercise (murmur, palpitation, or dyspnoea) or for a medical sports certificate will be assessed for eligibility. These children will be classified in the control group only after a completely normal check-up, including physical examination, electrocardiogram, and echocardiography. Children with any chronic disease, medical condition (cardiac, neurologic, respiratory, muscular, or renal), or medical treatment and those requiring any further specialized medical consultation will not be eligible.

Patients who are not able to answer the quality of life questionnaire (e.g. non-French speakers, severe mental illness) will not be eligible for the study. Detailed inclusion and exclusion criteria are reported in Table 1. 
Table 1

Trial entry.

\section{Inclusion criteria}

- Male or female aged 6 to 18 years old.

- Group 1: Patients with an inherited cardiac arrhythmia (long QT syndrome, Brugada syndrome, catecholaminergic polymorphic ventricular tachycardia, or arrhythmogenic right ventricular dysplasia), or those with an inherited cardiomyopathy (hypertrophic, dilated, or restrictive cardiomyopathy).

- Group 2: Children with a completely normal check-up, referred to the paediatric cardiology consultation for a non-severe functional symptom linked to exercise (murmur, palpitation, or dyspnoea) or for a medical sports certificate.

- Informed consent of parents or legal guardians, and oral assent of children.

\section{Exclusion criteria}

- Patients who are not able to understand or fill out the questionnaires (QoL, physical activity and motivation questionnaires).

- Absolute contraindications for CPET: fever, uncontrolled asthma, respiratory failure, acute myocarditis or pericarditis, uncontrolled arrhythmias causing symptoms or haemodynamic compromise, uncontrolled heart failure, acute pulmonary embolus or pulmonary infarction, and children with mental impairment leading to inability to cooperate.

- Group 2: Children with any chronic disease, medical condition (cardiac, neurologic, respiratory, muscular, or renal), or medical treatment and those requiring any further specialized medical consultation.

\subsection{Primary outcome}

The primary outcome is the total QoL score with the self-reported PedsQL questionnaire. The PedsQL questionnaire has four multidimensional scales: physical functioning ( 8 items), emotional functioning (5 items), social functioning (5 items), and school functioning (5 items). The three summary scores are: total scale score (23 items), physical health summary score (8 items), psychosocial health summary score (15 items). Each item uses a 5-point Likert scale from 0 (never) to 4 (almost always). Items are reversed scored and linearly transformed to a 0-100 scale, higher scores indicating a better QoL. This instrument was validated by Varni et al. in healthy and patient populations and its psychometric properties showed reliability, validity and responsiveness to clinical change over time [28]. The translation and cultural adaptation into French was performed by MAPI Research Institute (www.mapi-trust.org), following the international guidelines [28] and this version showed good psychometric properties [29]. Our group recently performed the complete psychometric validation of the French self and proxy versions of the PedsQL generic questionnaire for 8 to 12 year-old children [30]. We also found a good sensitivity of the PedsQL in several controlled prospective HRQoL studies among healthy controls and children with various chronic diseases [24-27].

In this study, three age versions of the PedsQL questionnaire will be used (e.g. 5-7, 8-12 and 13-18 years old). 


\subsection{Secondary outcomes}

- The level of physical activity will be assessed by:

The metabolic equivalent of task (MET), measured by a fitness tracker (ActiGraph GT3X accelerometer, Pensacola, FL, USA) [31]. The MET is defined as the ratio of physical activity to basal metabolic demand. The metabolic equivalence scale ranges from $0.9 \mathrm{MET}$ (at rest) to $18 \mathrm{MET}$ (run at $17.5 \mathrm{~km} / \mathrm{h}$ ). The participants will be instructed to wear the fitness tracker at the waist at all times for 14 days, except during sleep and water-based activities such as swimming or bathing.

The Ricci and Gagnon physical activity questionnaire, composed by 8 items (total score < 16 points: no activity; 17 to 32 points: moderate activity; 33 to 40 points: intensive activity) [32]. The EMAPS questionnaire (motivation scale for physical activity in a health context), a 18-item motivation scale towards health-oriented physical activity recently validated in French [33].

- The exercise capacity will be assessed by a cardio-pulmonary exercise test (CPET), using the same methodology as in our previous paediatric CPET studies [5, 20,21][34]. The following variables will be measured: maximum oxygen uptake ( $\left.\mathrm{VO} 2_{\max }\right)$; ventilatory anaerobic threshold (VAT); ventilatory efficiency (VE/VCO2 slope), oxygen uptake efficiency slope (OUES), and maximum oxygen pulse

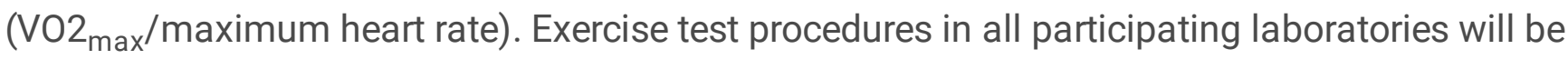
harmonized before the start of the study. All centres will use the same CPET cycle ergometer protocol, to obtain a homogeneous incremental overall duration between 8 to 12 minutes: a 1-minute rest; a 3-minute warm-up (10 to 20 watts) in increments of 10, 15, or 20 watts each minute; a pedalling rate of 60 to 80 revolutions per minute; a 3-minute active recovery (20 watts); and a 2minute rest. The CPET will be considered as maximal when 3 out of the 4 following criteria will be reached: respiratory exchange ratio $(\mathrm{RER}=\mathrm{VCO} 2 / \mathrm{VO} 2) \geq 1$, maximum heart rate $>85 \%$ of maximal age-predicted heart rate, limit of the patient's tolerance despite verbal encouragement, plateau of VO2 (VO2 ${ }_{\text {max }}$ ) despite the increasing exercise intensity, and patient's inability to provide a minimum pedalling frequency of 60 per minute despite verbal encouragement. The VAT will be estimated using Beaver's method [35]. $\mathrm{VO}_{\max }$ and VAT values will be normalized in a percentage of the predicted $\mathrm{VO} 2_{\max }$ using reference values for cycle ergometer test in the general paediatric population [36, 37]. When the $\mathrm{VO}_{\text {max }}$ does not reach criteria for maximal effort, the peak VO2 will be informed, as usual in paediatrics $[38,39]$.

- The clinical outcomes: age at diagnosis (prenatal, postnatal), genetic anomaly, NYHA functional class, blood pressure, body mass index (BMI), healthcare usage (primary and secondary care contacts, hospitalisation), pacemaker, implantable cardioverter defibrillator (ICD), cardiac surgery, and medication.

- The parents-reported quality of life score with the proxy version of the PedsQL (e.g. 5-7, 8-12 and 13-18 y.o. proxy versions) [28].

- The socio-economic status of the patient's family.

- The safety outcomes. 
All outcomes are reported in Table 2.

Table 2

Outcome measures.

\section{Primary outcome}

- Health-related quality of life score: PedsQL self-questionnaire (versions 5-7 years and 8-12 years for children and version 13-18 years for adolescents).

\section{Secondary outcomes}

- Level physical activity assessed by:

o The metabolic equivalent of task (MET) assessed by the ActiGraph GT3X accelerometer.

o The Ricci and Gagnon physical activity questionnaire.

o The EMAPS motivation questionnaire.

- CPET variables:

○ V02 $\max$ (maximum oxygen uptake).

o VAT (ventilatory anaerobic threshold).

o VE/VCO2 slope (ventilarory efficiency).

o OUES (oxygen uptake efficiency slope).

o Maximum oxygen pulse ( $\mathrm{VO} 2_{\max } /$ maximum heart rate).

- Clinical outcomes: age at diagnosis (prenatal, postnatal), genetic anomaly, NYHA functional class, blood pressure, body mass index (BMI), healthcare usage (primary and secondary care contacts, hospitalisation), pacemaker, implantable cardioverter defibrillator (ICD), cardiac surgery, and medication.

- Health-related quality of life score: PedsQL proxy questionnaire (versions 5-7 years and 8-12 years for children and version 13-18 years for adolescents).

- The socio-economic status of the family.

\subsection{Sample size}

We aim to recruit a total of 214 children in both groups. The primary outcome is the difference in the selfreported QoL score with the PedsQL instrument between the children with inherited cardiac disorders and the control group. We used the results from our previous QoL cross-sectional studies in patients with CHD to calculate the sample size [4, 21, 23-27]. In our experience, as well as in similar studies using patient related outcomes, a difference of less than 5 points is not clinically relevant, and a difference of more than 10 points is ideal, but rarely achieved $[2,25,27]$. Therefore, we hypothesized to observe a difference in the total QoL score of $7 \pm 15$ points (over 100 ). With a $90 \%$ power, a bilateral alpha risk of $5 \%$, and potentially $10 \%$ of loss to follow-up or missing data on the primary outcome, we need to include 107 patients in the group 1 and 107 patients in the group 2. 


\subsection{Statistical analysis}

Patients' characteristics will be presented using mean and standard deviation for continuous variables, and frequencies and proportions for qualitative variables. QoL scores will be calculated with the parametric Student's $t$ test when the distribution was Gaussian and with the Mann-Whitney test otherwise.

Association between QoL scores and physical activity, clinical and socio-demographic data will be studied using Pearson's or Spearman's coefficient. The statistical significance will be set at 0.05 and analyses will be performed using Statistical Analysis Systems version 9 (SAS Institute, Cary, NC, USA). Data will be managed by the clinical research unit of Montpellier University Hospital, France, in collaboration with the paediatric and congenital cardiology department.

\subsection{Ethics}

The study will be conducted in compliance with the Good Clinical Practices protocol and Declaration of Helsinki principles. It was approved by a drawn national Ethics Committee (CPP Sud-Est VI, 2020-A0041138). Informed consent will be obtained from all parents or legal guardians, and oral assent will be obtained from all children. The study was registered on Clinicaltrials.gov (NCT04712136).

\section{Expected Results And Perspectives}

In the continuity of our research program on QoL and physical activity in paediatric and congenital cardiology [4, 21-24, 24-27], the QUALIMYOTYRHM trial should provide, for the first time, reliable data on health-related QoL and physical activity in children with inherited cardiac arrhythmia or inherited cardiomyopathies. From our perspective, considering the QoL as the main outcome of this trial is both original and primordial in this young population with cardiac inherited disorders. Indeed, measuring patient-reported outcomes (PRO) in paediatric cardiology should become a priority, considering that many young cardiac patients experience a low level of physical impairment during childhood, but an increased cardio-vascular risk during adulthood [2, 3][40]. This is also in line with the European Medicines Agency (EMA) promotion of PRO measures in clinical research, and more and more drug trials, even in paediatrics, currently evaluate the quality of life as a secondary outcome [41]. Moreover, our results will contribute to provide consistent baseline clinical and QoL data for future interventional clinical trials in children with inherited cardiac arrhythmia or inherited cardiomyopathies.

The QUALIMYORYTHM trial also seeks to determine the level of physical activity in the contemporary generation of children with inherited cardiac disorders, using modern connected technology adapted to this population, such as fitness trackers. In the absence of any reliable risk stratification in those children, the current guidelines on eligibility of young athletes with inherited cardiac disorders for sports participation mainly rely on expert opinions [42-45], and conclusions by the experts have classically not been the same on both sides of the Atlantic [46]. The difference commonly made between recreational and competitive sport is probably more about medico-legal issues for the practitioner, than for the 
patient's own interest. For example, more than a decade ago, the European guidelines for competitive sport participation in the long QT syndrome were very restrictive [43]. Yet, in a recent study from a cohort of 172 appropriately managed children with long QT syndrome, cardiac event rates were low and occurred during recreational, but not competitive activities [47]. Similarly, in a cohort of 129 young athletes with ICD, most of them having a long QT syndrome or a hypertrophic cardiomyopathy, and participating in competitive or dangerous sports, there were no occurrences of death, arrest, or injury related to arrhythmia, during sports; interestingly, this study found that $27 \%$ patients received at least one shock, only 4 during sports, which is similar to unselected non-athletic paediatric ICD cohorts [48]. Based on convergent studies reporting a low risk of cardiac event in athletes with optimal treatment for long QT syndrome [47-49][50], a progressive trend towards a different medical approach has been observed, involving the patient-athlete in shared decision-making. Therefore, the 2015 US guidelines [51] have been less restrictive regarding high intensity recreational exercise and competitive sports, especially for asymptomatic genotype-positive/phenotype-negative athletes. More recently, the 2020 European guidelines have moved on step further in promoting supervised and regular physical activity in patients with inherited cardiac disorders [45].

Nevertheless, most published studies are retrospective and have not evaluated sports with the highest cardiovascular demand (only a few class IIIB and IIIC sports), or recreational sports in children with the rarest or most serious inherited cardiac disorders. Indeed, in their recent review, Masrur et al. concluded that there are insufficient data on the risks of exercise in Brugada syndrome to make recommendations for exercise [52]. Therefore, the QUALIMYORYTHM trial should contribute to precise how the recommendations are currently applied "in the real life", and measure their potential impact on QoL and physical activity of children with inherited cardiac arrhythmia or inherited cardiomyopathies.

Furthermore, the QUALIMYORYTHM trial will provide, for the first time in a population of children with inherited cardiac disorders, consistent cardiopulmonary fitness data, such as the maximum oxygen uptake $\left(\mathrm{VO}_{\mathrm{max}}\right)$, the anaerobic threshold, the ventilatory efficiency, and the exercise stroke volume. Moreover, this study will also bring original information about the level of motivation of children with inherited cardiac disorders for physical activity, and its degree of correlation with the actual levels of physical activity and exercise capacity. In paediatric cardiology, cardiopulmonary fitness has mainly been determined in children with $\mathrm{CHD}[5,53]$, but these results cannot be easily transposed to inherited cardiac disorders, as the underlying pathophysiology and haemodynamic consequences are different. For instance, animal models have demonstrated that the beneficial effects of training on the heart effects of training are related to the signalling pathways of myocardial hypertrophy and fibrosis, but such findings need to be further analysed in patients with inherited cardiomyopathies [54].

If the QUALIMYORYTHM trial finds that physical activity and exercise capacity in children with inherited cardiac arrhythmia or inherited cardiomyopathies are similar to those of healthy children, without any additional risk, the level of evidence for future guidelines on sports eligibility in this population will be significantly improved. Conversely, if physical activity and exercise capacity in children with inherited cardiac disorders are significantly impaired, it will open a new research field on rehabilitation programs in 
this population. Indeed, if physical deconditioning is diagnosed and managed at an early stage in patients with inherited cardiac disorders, participation in rehabilitation programs could reverse the vicious circle of deconditioning, and, ultimately participate in reducing the cardio-vascular risks related to inactivity, as it has been suggested in $\mathrm{CHD}[22,55-57]$, as well as in many chronic diseases [58].

\section{Abbreviations}

CHD: congenital heart disease

CPET: cardiopulmonary exercise test

QoL: health-related quality of life

\section{Declarations}

\section{Ethics approval and consent to participate}

The study will be conducted in compliance with the Good Clinical Practices protocol and Declaration of Helsinki principles. It was approved by a drawn national Ethics Committee (CPP Sud-Est VI, 2020-A0041138) and was registered on Clinicaltrials.gov (NCT04712136). Informed consent will be obtained from all parents or legal guardians, and oral assent will be obtained from all children.

\section{Consent for publication}

This study does not contain data from any individual person (images or videos).

\section{Availability of data and material}

Not applicable.

\section{Competing interests}

The Authors declare that they have no conflict of interest.

\section{Funding}

Montpellier university hospital is the sponsor of the QUALIMYORYHM trial. The study was funded after winning the 2019 call for proposal from the National Department of Health (DGOS-GIRCI-SOHO-APITHEM, www.girci-soho.fr) and the young researcher award from Montpellier University Hospital (AOI 2019). Additional research funding was obtained from the French Federation of Cardiology (2019 FFC "research team" call for proposal, www.fedecardio.org), and the national rare cardiovascular diseases network (2018 CARDIOGEN call for proposal, www.filiere-cardiogen.fr).

\section{Authors' contributions}


Study concept and design: PA

Drafting of the manuscript: $\mathrm{PA}, \mathrm{AH}, \mathrm{OW}$

Critical revision of the manuscript for important intellectual content: all

Statistical analysis: MCP

Obtained funding: PA, OW

Administrative, technical, or material support: HA

Study supervision: PA

\section{Acknowledgements}

The authors are grateful to CARDIOGEN for their financial support. We also thank Sophie Pierre and Lea Falloud, from the patient organisations "AMRYC" (association des patients atteints de maladies du rythme cardiaque), and "Ligue contre la cardiomyopathie", respectively, for their support.

\section{References}

1. Spector LG, Menk JS, Knight JH, McCracken C, Thomas AS, Vinocur JM. et al. Trends in Long-Term Mortality After Congenital Heart Surgery. J Am Coll Cardiol. 2018;71:2434-46.

2. Anker SD, Agewall S, Borggrefe M, Calvert M, Jaime Caro J, Cowie MR. et al. The importance of patient-reported outcomes: a call for their comprehensive integration in cardiovascular clinical trials. Eur Heart J. 2014;35:2001-9.

3. Moons P, Kovacs AH, Luyckx K, Thomet C, Budts W, Enomoto J. et al. Patient-reported outcomes in adults with congenital heart disease: Inter-country variation, standard of living and healthcare system factors. Int J Cardiol. 2018;251:34-41.

4. Amedro P, Picot MC, Moniotte S, Dorka R, Bertet H, Guillaumont S. et al. Correlation between cardiopulmonary exercise test variables and health-related quality of life among children with congenital heart diseases. Int J Cardiol. 2016;203:1052-60.

5. Amedro P, Gavotto A, Guillaumont S, Bertet H, Vincenti M. et al. Cardiopulmonary fitness in children with congenital heart diseases versus healthy children. Heart Br Card Soc. 2018;104:1026-36., De La Villeon G.

6. Takken T, Giardini A, Reybrouck T, Gewillig M, Hövels-Gürich HH, Longmuir PE. et al. Recommendations for physical activity, recreation sport, and exercise training in paediatric patients with congenital heart disease: a report from the Exercise, Basic \& Translational Research Section of the European Association of Cardiovascular Prevention and Rehabilitation, the European Congenital Heart and Lung Exercise Group, and the Association for European Paediatric Cardiology. Eur J Prev Cardiol. 2012;19:1034-65. 
7. Longmuir PE, Brothers JA. de Ferranti SD, Hayman LL, Van Hare GF, Matherne GP. et al. Promotion of physical activity for children and adults with congenital heart disease: a scientific statement from the American Heart Association. Circulation. 2013;127:2147-59.

8. Budts W, Pieles GE, Roos-Hesselink JW, Sanz de la Garza M, D’Ascenzi F, Giannakoulas G. et al. Recommendations for participation in competitive sport in adolescent and adult athletes with Congenital Heart Disease (CHD): position statement of the Sports Cardiology \& Exercise Section of the European Association of Preventive Cardiology (EAPC), the European Society of Cardiology (ESC) Working Group on Adult Congenital Heart Disease and the Sports Cardiology, Physical Activity and Prevention Working Group of the Association for European Paediatric and Congenital Cardiology (AEPC). Eur Heart J. 2020;41:4191-9.

9. Moola F, Fusco C, Kirsh JA. The perceptions of caregivers toward physical activity and health in youth with congenital heart disease. Qual Health Res. 2011;21:278-91.

10. Papasavvas T, Alhashemi M, Micklewright D. Association Between Depressive Symptoms and Exercise Capacity in Patients With Heart Disease: A META-ANALYSIS. J Cardiopulm Rehabil Prev. 2017;37:239-49.

11. Karsenty C, Maury P, Blot-Souletie N, Ladouceur M, Leobon B, Senac V. et al. The medical history of adults with complex congenital heart disease affects their social development and professional activity. Arch Cardiovasc Dis. 2015;108:589-97.

12. Tamayo C, Manlhiot C, Patterson K, Lalani S, McCrindle BW. Longitudinal evaluation of the prevalence of overweight/obesity in children with congenital heart disease. Can J Cardiol. 2015;31:117-23.

13. Moola F, McCrindle BW, Longmuir PE. Physical activity participation in youth with surgically corrected congenital heart disease: Devising guidelines so Johnny can participate. Paediatr Child Health. 2009;14:167-70.

14. Czosek RJ, Kaltman JR, Cassedy AE, Shah MJ, Vetter VL, Tanel RE. et al. Quality of Life of Pediatric Patients With Long QT Syndrome. Am J Cardiol. 2016;117:605-10.

15. Sleeper LA, Towbin JA, Colan SD, Hsu D, Orav EJ, Lemler MS, et al. Health-Related Quality of Life and Functional Status Are Associated with Cardiac Status and Clinical Outcome in Children with
Cardiomyopathy. J Pediatr. 2016;170:173-180.e1-4.

16. Gow RM, Borghese MM, Honeywell CR, Colley RC. Activity Intensity During Free-Living Activities in Children and Adolescents With Inherited Arrhythmia Syndromes: Assessment by Combined Accelerometer and Heart Rate Monitor. Circ Arrhythm Electrophysiol. 2013;6:939-45.

17. Hamang A, Eide GE, Nordin K, Rokne B, Bjorvatn C, Øyen N. Health status in patients at risk of inherited arrhythmias and sudden unexpected death compared to the general population. BMC Med Genet. 2010;11:27.

18. Rhodes AC, Murray B, Tichnell C, James CA, Calkins H, Sears SF. Quality of life metrics in arrhythmogenic right ventricular cardiomyopathy patients: The impact of age, shock and sex. Int $\mathrm{J}$ Cardiol. 2017;248:216-20. 
19. Christian S, Somerville M, Taylor S, Spence JC, Giuffre M, Atallah J. The impact of physical activity modification on the well-being of a cohort of children with an inherited arrhythmia or cardiomyopathy. Cardiol Young. 2020;30:692-7.

20. Gavotto A, Vandenberghe D, Abassi H, Huguet H, Macioce V, Picot M-C. et al. Oxygen uptake efficiency slope: a reliable surrogate parameter for exercise capacity in healthy and cardiac children? Arch Dis Child. 2020;105:1167-74.

21. Abassi H, Gavotto A, Picot MC, Bertet H, Matecki S, Guillaumont S. et al. Impaired pulmonary function and its association with clinical outcomes, exercise capacity and quality of life in children with congenital heart disease. Int J Cardiol. 2019;285:86-92.

22. Amedro P, Gavotto A, Legendre A, Lavastre K, Bredy C. et al. Impact of a centre and home-based cardiac rehabilitation program on the quality of life of teenagers and young adults with congenital heart disease: The QUALI-REHAB study rationale, design and methods. Int J Cardiol. 2019;283:1128., De La Villeon G.

23. Amedro P, Dorka R, Moniotte S, Guillaumont S, Fraisse A, Kreitmann B. et al. Quality of Life of Children with Congenital Heart Diseases: A Multicenter Controlled Cross-Sectional Study. Pediatr Cardiol. 2015;36:1588-601.

24. Amedro P, Tahhan N, Bertet H, Jeandel C, Guillaumont S, Mura T. et al. Health-related quality of life among children with Turner syndrome: controlled cross-sectional study. J Pediatr Endocrinol Metab JPEM. 2017;30:863-8.

25. Amedro P, Bajolle F, Bertet $H$, Cheurfi R, Lasne D, Nogue E. et al. Quality of life in children participating in a non-selective INR self-monitoring VKA-education programme. Arch Cardiovasc Dis. 2018;111:180-8.

26. Abassi H, Bajolle F, Werner O, Auer A, Marquina A, Mura T. et al. Health-related quality of life correlates with time in therapeutic range in children on anticoagulants with International Normalised Ratio self-monitoring. Arch Cardiovasc Dis. 2020.

27. Abassi H, Huguet H, Picot M-C, Vincenti M, Guillaumont S, Auer A. et al. Health-related quality of life in children with congenital heart disease aged 5 to 7 years: a multicentre controlled cross-sectional study. Health Qual Life Outcomes. 2020;18:366.

28. Varni JW, Seid M, Kurtin PS. PedsQL 4.0: reliability and validity of the Pediatric Quality of Life Inventory version 4.0 generic core scales in healthy and patient populations. Med Care. 2001;39:800-12.

29. Tessier S, Vuillemin A, Lemelle J-L, Briançon S. Propriétés psychométriques du questionnaire générique français « Pediatric Quality of Life Inventory Version 4.0» (PedsQLTM 4.0). Eur Rev Appl Psychol. 2009;59:291-300.

30. Amedro P, Huguet $\mathrm{H}$, Macioce V, Dorka R, Auer A, Guillaumont S. et al. Psychometric validation of the French self and proxy versions of the PedsQL ${ }^{\text {T' }} 4.0$ generic health-related quality of life questionnaire for 8-12 year-old children. Health Qual Life Outcomes. 2021;19:75. 
31. Cunningham C, Spence JC, Stearns JA, Carson V, Kantor PF, Urschel S. et al. Self-reported and Accelerometer-Measured Physical Activity in Children With Cardiomyopathy. J Cardiovasc Nurs. 2020;35:300-6.

32. Vuillemin A, Denis G, Guillemin F, Jeandel C. [A review of evaluation questionnaires for physical activity]. Rev Epidemiol Sante Publique. 1998;46:49-55.

33. Boiché J, Gourlan M, Trouilloud D, Sarrazin P. Development and validation of the "Echelle de Motivation envers I'Activité Physique en contexte de Santé": A motivation scale towards healthoriented physical activity in French. J Health Psychol. 2019;24:386-96.

34. Gavotto A, Huguet H, Picot M-C, Guillaumont S, Matecki S, Amedro P. The Ve/Vco2 slope: a useful tool to evaluate the physiological status of children with congenital heart disease. J Appl Physiol Bethesda Md 1985. 2020;129:1102-10.

35. Beaver WL, Wasserman K, Whipp BJ. A new method for detecting anaerobic threshold by gas exchange. J Appl Physiol Bethesda Md 1985. 1986;60:2020-7.

36. Cooper DM, Berry C, Lamarra N, Wasserman K. Kinetics of oxygen uptake and heart rate at onset of exercise in children. J Appl Physiol Bethesda Md 1985. 1985;59:211-7.

37. Cooper DM, Weiler-Ravell D, Whipp BJ, Wasserman K. Aerobic parameters of exercise as a function of body size during growth in children. J Appl Physiol. 1984;56:628-34.

38. Barker AR, Williams CA, Jones AM, Armstrong N. Establishing maximal oxygen uptake in young people during a ramp cycle test to exhaustion. Br J Sports Med. 2011;45:498-503.

39. Rowland TW, Cunningham LN. Oxygen uptake plateau during maximal treadmill exercise in children. Chest. 1992;101:485-9.

40. Saha P, Potiny P, Rigdon J, Morello M, Tcheandjieu C, Romfh A. et al. Substantial Cardiovascular Morbidity in Adults With Lower-Complexity Congenital Heart Disease. Circulation. 2019;139:188999.

41. Apolone G. De Carli G, Brunetti M, Garattini S. Health-related quality of life (HR-QOL) and regulatory issues. An assessment of the European Agency for the Evaluation of Medicinal Products (EMEA) recommendations on the use of HR-QOL measures in drug approval. PharmacoEconomics. 2001;19:187-95.

42. Maron BJ, Chaitman BR, Ackerman MJ, Bayés de Luna A, Corrado D, Crosson JE. et al. Recommendations for physical activity and recreational sports participation for young patients with genetic cardiovascular diseases. Circulation. 2004;109:2807-16.

43. Pelliccia A, Fagard R, Bjørnstad HH, Anastassakis A, Arbustini E, Assanelli D. et al. Recommendations for competitive sports participation in athletes with cardiovascular disease: a consensus document from the Study Group of Sports Cardiology of the Working Group of Cardiac Rehabilitation and Exercise Physiology and the Working Group of Myocardial and Pericardial Diseases of the European Society of Cardiology. Eur Heart J. 2005;26:1422-45.

44. Priori SG, Blomström-Lundqvist C, Mazzanti A, Blom N, Borggrefe M, Camm J. et al. 2015 ESC Guidelines for the management of patients with ventricular arrhythmias and the prevention of 
sudden cardiac death: The Task Force for the Management of Patients with Ventricular Arrhythmias and the Prevention of Sudden Cardiac Death of the European Society of Cardiology (ESC). Endorsed by: Association for European Paediatric and Congenital Cardiology (AEPC). Eur Heart J. 2015;36:2793-867.

45. Pelliccia A, Sharma S, Gati S, Bäck M, Börjesson M, Caselli S. et al. 2020 ESC Guidelines on sports cardiology and exercise in patients with cardiovascular disease. Eur Heart J. 2020.

46. Schnell F, Behar N, Carré F. Long-QT Syndrome and Competitive Sports. Arrhythmia Electrophysiol Rev. 2018;7:187-92.

47. Chambers KD, Beausejour Ladouceur V, Alexander ME, Hylind RJ, Bevilacqua L, Mah DY. et al. Cardiac Events During Competitive, Recreational, and Daily Activities in Children and Adolescents With Long QT Syndrome. J Am Heart Assoc. 2017;6.

48. Vickers SE, Ian L, Berul Charles I, Ackerman MJ, Kanter Ronald J, Shubhayan S. et al. Safety of Sports for Young Patients With Implantable Cardioverter-Defibrillators. Circ Arrhythm Electrophysiol American Heart Association. 2018;11:e006305.

49. Aziz PF, Saarel EV. Sports participation in long QT syndrome. Cardiol Young. 2017;27:43-8.

50. Johnson JN, Ackerman MJ. Return to play? Athletes with congenital long QT syndrome. Br J Sports Med. 2013;47:28-33.

51. Ackerman MJ, Zipes DP, Kovacs RJ, Maron BJ. Eligibility and Disqualification Recommendations for Competitive Athletes With Cardiovascular Abnormalities: Task Force 10: The Cardiac Channelopathies: A Scientific Statement From the American Heart Association and American College of Cardiology. J Am Coll Cardiol. 2015;66:2424-8.

52. Masrur S, Memon S, Thompson PD. Brugada syndrome, exercise, and exercise testing. Clin Cardiol. 2015;38:323-6.

53. Müller J, Böhm B, Semsch S, Oberhoffer R, Hess J, Hager A. Currently, children with congenital heart disease are not limited in their submaximal exercise performance. Eur $\mathrm{J}$ Cardio-Thorac Surg Off $\mathrm{J}$ Eur Assoc Cardio-Thorac Surg. 2013;43:1096-100.

54. Kyselovič J, Leddy JJ. Cardiac Fibrosis: The Beneficial Effects of Exercise in Cardiac Fibrosis. Adv Exp Med Biol. 2017;999:257-68.

55. Rhodes J, Curran TJ, Camil L, Rabideau N, Fulton DR, Gauthier NS. et al. Sustained effects of cardiac rehabilitation in children with serious congenital heart disease. Pediatrics. 2006;118:e586-93.

56. Gomes-Neto M, Saquetto MB. da Silva e Silva CM, Conceição CS, Carvalho VO. Impact of Exercise Training in Aerobic Capacity and Pulmonary Function in Children and Adolescents After Congenital Heart Disease Surgery: A Systematic Review with Meta-analysis. Pediatr Cardiol. 2016;37:217-24.

57. Amedro P, Gavotto A, Bredy C, Guillaumont S. [Cardiac rehabilitation for children and adults with congenital heart disease]. Presse Medicale Paris Fr 1983. 2017;46:530-7.

58. Booth FW, Roberts CK, Laye MJ. Lack of exercise is a major cause of chronic diseases. Compr Physiol. 2012;2:1143-211. 